\title{
Entanglement in a first-order quantum phase transition
}

\author{
Julien Vidal, ${ }^{1, *}$ Rémy Mosseri, ${ }^{1, \dagger}$ and Jorge Dukelsky ${ }^{2, \pitchfork}$ \\ ${ }^{1}$ Groupe de Physique des Solides, CNRS UMR 7588, Campus Boucicaut, 140 rue de Lourmel, 75015 Paris, France \\ ${ }^{2}$ Instituto de Estructura de la Materia, CSIC, Madrid, Spain
}

(Received 4 December 2003; published 12 May 2004)

\begin{abstract}
We analyze the entanglement properties of the ground state for a system of spins half embedded in a magnetic field, mutually interacting antiferromagnetically. Contrary to the ferromagnetic case where a secondorder quantum phase transition occurs, a first-order transition is obtained at zero field and the so-called concurrence which measures the two-spin entanglement displays a jump at the transition point.
\end{abstract}

DOI: 10.1103/PhysRevA.69.054101

One of the most fascinating features of the quantum world is certainly the entanglement, which has no classical counterpart. Celebrated by the pioneering works of Schrödinger [1] and Einstein, Podolsky, and Rosen [2] about the nonlocality, ubiquitous in the field of quantum information [3-5], entanglement properties of quantum systems have recently attracted much attention in the context of phase transitions. In the various models studied, such as spin chains in a transverse magnetic field [6-12], spin ladders [13], spin simplex $[14,15]$, and the Hubbard model [16], the ground state entanglement has been shown to be strongly modified at the critical point raising the question of the universality of these behaviors. Indeed, if the scaling of the entanglement seems to be universal, its range depends on the model [7]. More surprisingly, even in a second-order quantum phase transition, the entanglement as measured by the so-called concurrence which, roughly speaking, measures the two-spin quantum correlations [17], is not always a smooth function of the external parameters that drive the transition [14]. Thus, further investigations are clearly required, especially in dimensions larger than one where most of the calculations have, up to now, been performed. Unfortunately, in two or three dimensions, very few models are exactly solvable and numerical diagonalizations are often restricted to a small number of degrees of freedom (typically, a few tenths), so that the thermodynamical limit is difficult to study.

In this paper, we consider a system where $N$ spins half embedded in a magnetic field $h$ mutually interact. We focus here on the antiferromagnetic case, the ferromagnetic one being discussed in Ref. [14]. The symmetries of the Hamiltonian allow us to considerably simplify its diagonalization and to determine the phase diagram in the thermodynamical limit. A first-order quantum phase transition is found at zero field whereas in the ferromagnetic case, a second-order transition occurs at a nonvanishing field. Next, we study the entanglement properties of the ground state via the concurrence computed from the thermal density matrix at zero temperature. This concurrence, which is nontrivial for $h>0$, is shown to be discontinuous at the transition point where it switches to zero.

\footnotetext{
*Electronic address: vidal@gps.jussieu.fr

${ }^{\dagger}$ Electronic address: mosseri@gps.jussieu.fr

‡Electronic address: dukelsky@iem.cfmac.csic.es
}

PACS number(s): 03.65.Ud, 03.67.Mn, 73.43.Nq

We consider the following Hamiltonian which generalizes the model introduced in Ref. [18]:

$$
\begin{aligned}
H & =-\frac{\lambda}{N} \sum_{i<j}\left(\sigma_{x}^{i} \sigma_{x}^{j}+\gamma \sigma_{y}^{i} \sigma_{y}^{j}\right)-h \sum_{i} \sigma_{z}^{i} \\
& =-\frac{2 \lambda}{N}\left(S_{x}^{2}+\gamma S_{y}^{2}\right)-2 h S_{z}+\frac{\lambda}{2}(1+\gamma),
\end{aligned}
$$

where the $\sigma_{\alpha}$ 's are the Pauli matrices and $S_{\alpha}=\Sigma_{i} \sigma_{\alpha}^{i} / 2$. The prefactor $1 / N$ is necessary to get a finite free energy per spin in the thermodynamical limit. Without loss of generality, we will set $h \geqslant 0$ in the following. The Hamiltonian $H$ preserves the magnitude of the total spin and does not couple states having a different parity of the number of spins pointing in the magnetic field direction (spin-flip symmetry), namely,

$$
\begin{gathered}
{\left[H, \mathbf{S}^{2}\right]=0,} \\
{\left[H, \prod_{i} \sigma_{z}^{i}\right]=0,}
\end{gathered}
$$

for all anisotropy parameter $\gamma$. In addition, it is straightforward to show that the full spectrum of $H$ is odd under the transformation $\lambda \rightarrow-\lambda$ and even under $h \rightarrow-h$. Furthermore, since $H$ writes in terms of the total spin operators, the degeneracy of each eigenvalue belonging to a spin $S$ sector is at least equal to the number of spin $S$ representations which is simply given by

$$
D_{S}=\left(\begin{array}{c}
N \\
N / 2-S
\end{array}\right)-\left(\begin{array}{c}
N \\
N / 2-S-1
\end{array}\right)
$$

for all $N$. This implies that the full spectrum is obtained by diagonalizing only one representation of each spin sector $S$ which allows us to deal with a large number of spins. Denoting by $\{|S, M\rangle\}$ an eigenbasis of $\mathbf{S}^{2}$ and $S_{z}$, the matrix elements of $H$ reads

$$
\begin{aligned}
\left\langle S^{\prime}, M^{\prime}|H| S, M\right\rangle= & \delta_{S, S^{\prime}}\left\{\left[-\frac{\lambda}{N}(1+\gamma)\left(S(S+1)-M^{2}-N / 2\right)\right.\right. \\
& -2 h M] \delta_{M^{\prime}, M}-\frac{\lambda(1-\gamma)}{2 N}\left(a_{M-1}^{S^{-}} a_{M}^{S^{-}} \delta_{M^{\prime}, M-2}\right. \\
& \left.\left.+a_{M+1}^{S^{+}} a_{M}^{S^{+}} \delta_{M^{\prime}, M+2}\right)\right\},
\end{aligned}
$$




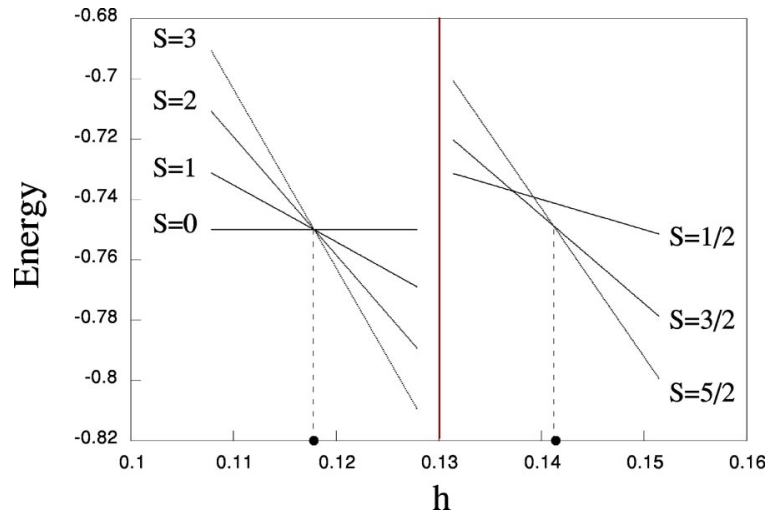

FIG. 1. Ground state energy of each spin sector as a function of the magnetic field for $\gamma=1 / 2(\lambda=-1)$. In the even case $N=6$ (left) all levels degenerate at $h_{\text {susy }}(\bullet)$, whereas in the odd one $N=5$ (right) a cascade is observed from the $S=N / 2$ to the $S=1 / 2$ sector.

where $a_{M}^{S^{ \pm}}=\sqrt{S(S+1)-M(M \pm 1)}$. These expressions, which are valid for any value of the parameters $(\lambda, h, \gamma)$ and for any $N$, generalize to any spin sector those given in Ref. [14] for the Dicke subspace $(S=N / 2)$. In the isotropic case $\gamma=1$, one further has $\left[H, S_{z}\right]=0$ so that $H$ is diagonal in the basis $\{|S, M\rangle\}$.

The antiferromagnetic nature of the coupling between spins considered here $(\lambda<0)$ completely modifies the phase diagram of $H$ as compared to the ferromagnetic case. A simple mean-field approach analogous to the one presented in Refs. $[19,20]$ can be performed and predicts a first-order phase transition in the zero field limit for any positive $\gamma$. The magnetization (per spin) in the $z$ direction of the ground state is given for all $\gamma \geqslant 0$ by

$$
\frac{1}{N}\left\langle S_{z}\right\rangle=\frac{1}{2} \operatorname{sgn}(h)
$$

where sgn denotes the signum function which vanishes for $h=0$. In the thermodynamical limit, the ground state is thus the fully polarized state for $h \neq 0$. Contrary to the ferromagnetic case, the ground state for a finite arbitrary $N$ does not, a priori, lie in the symmetric representation of the permutation group spanned by the Dicke states [14]. Indeed, in the zero field limit $(h \rightarrow 0)$, the ground state shall belong to the lowest spin sector that minimizes the interaction term. The main issue is thus to determine the values of $h$ for which level crossings appears.

For illustration, we have displayed in Fig. 1 the ground state energy of the different spin sector for a small number of spins. Two different scenarios arise according to the parity of $N$. In the even $N$ case an additional symmetry allows us to give a complete description of the ground state properties. Indeed, as recently pointed out by Unanyan and Fleischhauer [21], when

$$
\left|h_{\text {susy }}\right|=\frac{|\lambda| \sqrt{\gamma}}{N}
$$

the Hamiltonian is supersymmetric [22]. When the supersymmetric (SUSY) condition (8) is fulfilled for an antiferro- magnetic interaction $(\lambda<0)$, the authors of Ref. [21] claim that the ground state is nondegenerate and given by

$$
\left|\psi_{0}\right\rangle=A e^{-\eta S_{z}}\left|N / 2,0_{y}\right\rangle,
$$

where $A$ is a normalization constant and where $\left|N / 2,0_{y}\right\rangle$ denotes the eigenstate of $\mathbf{S}^{2}$ and $S_{y}$ with eigenvalues $(N / 2)(N / 2+1)$ and 0 , respectively. The parameter $\eta$ is determined by the relation [23]: tanh $\eta=\lambda / N$. Even though $\left|\psi_{0}\right\rangle$ is a ground state of $H$ with total spin $S=N / 2$, the spectrum at the SUSY point is, however, highly degenerate. Indeed, at the SUSY point $h_{\text {susy }}$, all the lowest eigenvalues of each spin representation are equal $\left[E_{0}=\lambda(1\right.$ $+\gamma) / 2]$ and the degeneracy of the ground state is thus given by

$$
d_{g}\left(\lambda=\lambda_{\text {susy }}\right)=\sum_{S=0}^{N / 2} D_{S}=\left(\begin{array}{c}
N \\
N / 2
\end{array}\right) .
$$

This collapse of the spectrum can be easily analyzed in the isotropic case $\gamma=1$ for which any state $|S, M\rangle$ is an eigenstate with an eigenvalue

$$
E(S, M)=-\frac{2 \lambda}{N}\left[S(S+1)-M^{2}\right]+\lambda-2 h M .
$$

For $\lambda<0$ and $h>0, E(S, M)$ is minimum for $M=S$ and at the SUSY point $E(S, S)=\lambda$ for any $S$.

The existence of this supersymmetric point enables us to locate the ground state for any value of the parameters. Indeed, $H$ describes a competition between the magnetic field $h$, which aims to align the spin in the field direction, and the interaction term which favors antiferromagnetic configurations. Thus, since at the SUSY point there exists a ground state lying in the maximum spin sector $S=N / 2$, the ground state for $h>h_{\text {susy }}$ also lies in this sector and $d_{g}\left(h>h_{\text {susy }}\right)$ $=D_{N / 2}=1$. Similarly, since singlet states $|0,0\rangle$ are also ground states at the SUSY point, they remain ground states for $h<h_{\text {susy }}$ and $d_{g}\left(h<h_{\text {susy }}\right)=D_{0}$. In the thermodynamical limit $(N \rightarrow \infty), h_{\text {susy }}$ goes to zero and the level crossing between $S=N / 2$ and $S=0$ ground states thus occurs at zero field. Nevertheless, for $h=0$, it is clear that the ground states have a zero total spin for all $\gamma \geqslant 0$.

For odd $N$, the situation is more complex since the ground states of each spin sector do not degenerate as in the even $N$ case, except in the thermodynamical limit. In fact, as previously shown the ground state belongs to the $S=N / 2$ sector for $h>h_{\text {susy }}$, and then switches to the other spin sector with decreasing $S$ when $h$ is lowered below $h_{\text {susy }}$. Naturally, for $h=0$, the ground state lies in the minimum spin sector and is given by all states $|1 / 2,1 / 2\rangle$. Its degeneracy $D_{S}$ thus strongly depends on $h$. Nevertheless, in the thermodynamical limit, the region in which these level crossings occurs shrinks and converges to the zero field point so that the parity of $N$ becomes irrelevant for the macroscopic physical quantities.

To analyze the entanglement properties of the ground state, we focus on the concurrence $C$ which has been introduced by Wootters [17] to measure the two-spin entanglement. This quantity is obtained from the density matrix de- 
scribing the state to be characterized. Here, we concentrate on the ground state of $H$ that can, as explained above, be degenerate. Thus, we must consider the thermal density matrix (at zero temperature) defined by

$$
\rho_{\text {th. }}=\frac{1}{d_{g}} \sum_{i=1}^{d_{g}}\left|\psi_{i}\right\rangle\left\langle\psi_{i}\right|,
$$

where $d_{g}$ is the degeneracy of the ground state and where $\left\{\left|\psi_{i}\right\rangle, i=1, \ldots, d_{g}\right\}$ constitutes an orthogonal basis of the $d_{g}$-dimensional lowest energy subspace $\mathcal{E}_{0}$. Indeed, if we would consider the projector onto a specific state belonging to $\mathcal{E}_{0}$, the entanglement properties would strongly depend on this choice [6]. Then, let $\rho$ be the reduced density matrix obtained by tracing out $\rho_{\text {th }}$ over $(N-2)$ spins. Of course, in our system, the choice of the two spins kept is irrelevant because of the permutation symmetry. Next, we introduce the spin-flipped density matrix $\tilde{\rho}=\sigma_{y} \otimes \sigma_{y} \rho^{*} \sigma_{y} \otimes \sigma_{y}$, where $\rho^{*}$ is the complex conjugate of $\rho$. The concurrence $C$ is then defined by

$$
C=\max \left\{0, \mu_{1}-\mu_{2}-\mu_{3}-\mu_{4}\right\},
$$

where the $\mu_{j}$ are the square roots of the four real eigenvalues of $\rho \tilde{\rho}$, classified in decreasing order. This concurrence vanishes for an unentangled two-body state whereas $C=1$ for a maximally entangled one. As explained in Ref. [14], it is further important to deal with a rescaled concurrence $C_{R}$ $=(N-1) C$ to take into account the coordination number of each spin. For a large number of spins, the difficulty comes from the trace step which requires operation in the full Hilbert space which is $2^{N}$ dimensional.

For $h>h_{\text {susy }}$, this can be achieved since the ground state lies in the sector $S=N / 2$. Indeed, (i) the $S=N / 2$ subspace is nondegenerate so that the thermal density matrix of the unique ground state [24] $|\psi\rangle=\Sigma_{M} \alpha_{M}|N / 2, M\rangle$ simply writes $\rho_{\text {th }}=|\psi\rangle\langle\psi|$, and (ii) the symmetry of the Dicke states $|N / 2, M\rangle[25]$ allows us to write down in a simple form the reduced density matrix $\rho$ in the standard basis $\{|\uparrow \uparrow\rangle,|\uparrow \downarrow\rangle,|\downarrow \uparrow\rangle,|\downarrow \downarrow\rangle\}$. One has [26]

$$
\begin{gathered}
\rho_{11}=\sum_{M}\left|\alpha_{M}\right|^{2} \frac{(N+2 M)(N+2 M-2)}{4 N(N-1)}, \\
\rho_{22}=\sum_{M}\left|\alpha_{M}\right|^{2} \frac{(N-2 M)(N+2 M)}{4 N(N-1)}, \\
\rho_{44}=\sum_{M}\left|\alpha_{M}\right|^{2} \frac{(N-2 M)(N-2 M-2)}{4 N(N-1)}, \\
\rho_{14}=\sum_{M} \alpha_{M} \alpha_{M+2}^{*} \sqrt{(N+2 M)(N+2 M-2)} \\
\quad \times \frac{\sqrt{(N-2 M+2)(N-2 M+4)}}{4 N(N-1)} .
\end{gathered}
$$

Furthermore, one has $\rho_{23}=\rho_{33}=\rho_{22}$, and $\rho^{*}=\rho$. The other matrix elements can also be computed for an arbitrary

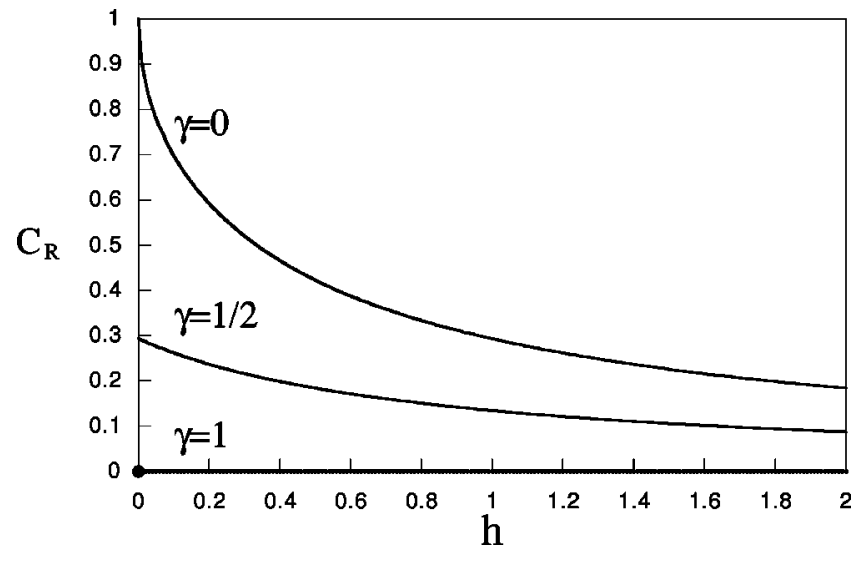

FIG. 2. Rescaled concurrence of the ground state as a function of the magnetic field for various anisotropy parameter $\gamma$ and for $N=10^{3}$ spins $(\lambda=-1)$. Note that for any $\gamma$, one has $C_{R}=0$ at zero field.

state belonging to $S=N / 2$ subspace but since they vanish for the eigenstates of $H$ because of Eq. (4), we do not give them here. Note that for the Dicke states, one recovers the expressions given in Refs. [26,27].

For $h<h_{\text {susy }}$, the ground state lies in the minimum spin sector and is highly degenerate. The thermal density matrix is then simply the projector onto the subspace corresponding to $S=0$ for even $N$ and $S=1 / 2$ for odd $N$. The concurrence computed from such a density matrix is known to vanish for $N>3$ (see Ref. [27]).

Figure 2 shows the behavior of the rescaled concurrence of the ground state as a function of the magnetic field. Note that for $\gamma=1$ and $h>h_{\text {susy }}$ the ground state is simply the Dicke state $|N / 2, N / 2\rangle$ for which $C=0$. The same result also trivially holds in the large $h$ limit for any $\gamma$.

For $h=h_{\text {susy }}$ the worst situation is reached, at least for even $N$, since there is one ground state in each spin sector. As a consequence, the thermal density matrix is a sum of projectors onto states of very different natures so that the trace operation is a rather difficult task for large $N$. Here, we have chosen to focus on $\left|\psi_{0}\right\rangle$ whose analytic expression (9) allows one to compute its concurrence for any even $N$. The coefficients $\alpha_{M}$ entering in its decomposition onto the Dicke states are simply given by

$$
\alpha_{N / 2-2 j}=e^{-\eta(N / 2-2 j)}\left(\begin{array}{c}
N / 2 \\
j
\end{array}\right) /\left(\begin{array}{l}
N \\
2 j
\end{array}\right)^{1 / 2},
$$

where we have set $M=N / 2-2 j$ because of the symmetry $\Pi_{i} \sigma_{z}^{i}\left|\psi_{0}\right\rangle=\left|\psi_{0}\right\rangle$. Two limiting cases can be easily analyzed: the $X Y$ case $(\gamma=1)$ for which $C_{R}=0$, and the Ising model $(\gamma=0)$ for which $\eta=0$ and $C_{R}=1$. The rescaled concurrence of $\left|\psi_{0}\right\rangle$ as a function of $\gamma$ is displayed in Fig. 3 .

In the large $N$ limit, the behavior of the rescaled concurrence of $\left|\psi_{0}\right\rangle$ can be computed and is given by

$$
C_{R}(\gamma)=1-\sqrt{\gamma}
$$

for $0 \leqslant \gamma \leqslant 1$. Of course, this information does not enable us to conclude anything about the concurrence computed with 


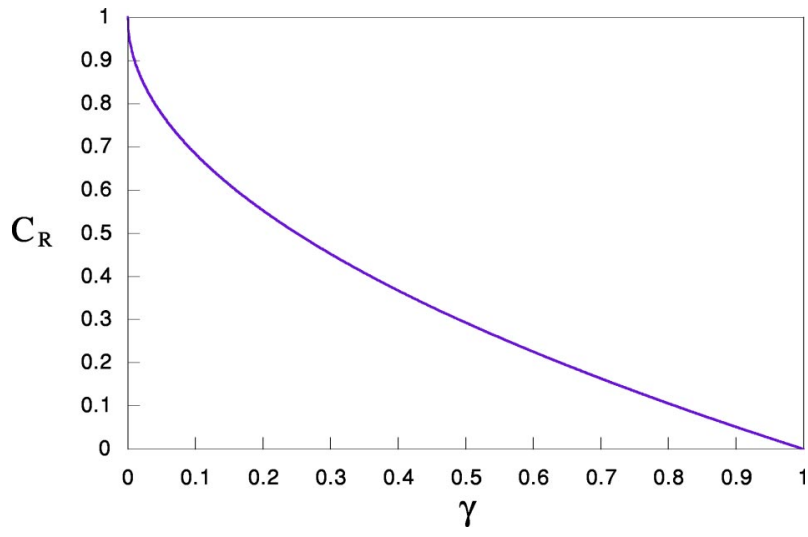

FIG. 3. Rescaled concurrence of the ground state $\left|\psi_{0}\right\rangle$ at the SUSY point as a function of the anisotropy parameter $\gamma$ for $N$ $=10^{4}$ spins.

the full thermal density matrix involving all spin sectors but it certainly points out a nontrivial behavior of the true ground state at $h=h_{\text {susy }}^{+}$.

Such a discontinuity of the concurrence at the transition point has already been obtained in other frustrated spin models, such as spin ladders or Heisenberg antiferromagnets in the Kagomé lattice [13]. However, in these systems the ground state entanglement properties are very simple (valence-bond-like states) so that its concurrence is constant in each region of the phase diagram. If a jump of the ground state concurrence seems reasonable for a system undergoing a first-order quantum phase transition, it is not obvious that other measures of the entanglement would have shown discontinuity. More precisely, in the above-mentioned example as well as in our model, the concurrence is found to become trivial for some parameter values.

An interesting perspective would be to study other measures of the entanglement in systems undergoing a quantum phase transition, such as the $N$ tangle [28] or the Minkovskian-square norm of the Stokes tensor [29] which investigate the $N$-spin entanglement. Indeed, the trace operation performed in the concurrence calculation undoubtedly kills some correlations between spins that could be captured by other types of measurement. One may also wonder how the entanglement would be affected if one changes the range of the interaction. This could be achieved, for example, by considering a one-dimensional system with periodic boundary conditions and long-range interactions. Such a model would interpolate between the one-dimensional Ising-like model for short-ranged interactions (nearest neighbors) and our model in the (constant) infinite-range limit.

We are indebted to B. Douçot and D. Mouhanna for fruitful and valuable discussions.
[1] E. Schrödinger, Naturwissenschaften 23, 807 (1935); 23, 823 (1935); 23, 844 (1935).

[2] A. Einstein, B. Podolsky, and N. Rosen, Phys. Rev. 47, 777 (1935).

[3] J. Preskill, J. Mod. Opt. 47, 127 (2000).

[4] D. Bouwmeester, A. Eckert, and A. Zeilinger, The Physics of Quantum Information (Springer-Verlag, Berlin, 2000).

[5] M. A. Nielsen and I. L. Chuang, Quantum Computation and Quantum Information (Cambridge University Press, Cambridge, 2000).032307

[6] T. J. Osborne and M. A. Nielsen, Phys. Rev. A 66, 032110 (2002).

[7] A. Osterloh, L. Amico, G. Falci, and R. Fazio, Nature (London) 416, 608 (2002).

[8] G. Vidal, J. I. Latorre, E. Rico, and A. Kitaev, Phys. Rev. Lett. 90, 227902 (2003).

[9] J. I. Latorre, E. Rico, and G. Vidal, e-print quant-ph/0304098.

[10] S. J. Gu, H. Q. Lin, and Y. Q. Li, Phys. Rev. A 68, 042330 (2003).

[11] U. Glaser, H. Büttner, and H. Fehske, Phys. Rev. A 68, 032318 (2003).

[12] V. E. Korepin, Phys. Rev. Lett. 92, 096402 (2004).

[13] I. Bose and E. Chattopadhyay, Phys. Rev. A 66, 062320 (2002).
[14] J. Vidal, G. Palacios, and R. Mosseri, Phys. Rev. A 69, 022107 (2004).

[15] N. Lambert, C. Emary, and T. Brandes, Phys. Rev. Lett. 92, 073602 (2004)

[16] S. J. Gu, Y. Q. Li, and H. Q. Lin, e-print quant-ph/0310030.

[17] W. K. Wootters, Phys. Rev. Lett. 80, 2245 (1998).

[18] H. J. Lipkin, N. Meshkov, and A. J. Glick, Nucl. Phys. 62, 188 (1965).

[19] R. Botet, R. Jullien, and P. Pfeuty, Phys. Rev. Lett. 49, 478 (1982).

[20] R. Botet and R. Jullien, Phys. Rev. B 28, 3955 (1983).

[21] R. G. Unanyan and M. Fleischhauer, Phys. Rev. Lett. 90, 133601 (2003).

[22] E. Witten, Nucl. Phys. B 188, 513 (1981).

[23] Note that a sign error has been inserted in Ref. [21].

[24] Contrary to the ferromagnetic case where it can be doubly degenerate [14], the ground state in the $S=N / 2$ sector is always nondegenerate.

[25] R. H. Dicke, Phys. Rev. 93, 99 (1954).

[26] J. K. Stockton, J. M. Geremia, A. C. Doherty, and H. Mabuchi, Phys. Rev. A 67, 022112 (2003).

[27] X. Wang and K. Mølmer, Eur. Phys. J. D 18, 385 (2002).

[28] A. Wong and N. Christensen, Phys. Rev. A 63, 044301 (2001).

[29] G. Jaeger et al., Phys. Rev. A 67, 032307 (2003). 